\title{
Triplet state formation and cis $\rightarrow$ trans isomerization in the excited singlet state of the keto tautomer of 2-(2'-hydroxyphenyl) benzothiazole
}

\author{
Wajih Al-Soufi, Karl H. Grellmann and Bernhard Nickel \\ Max-Planck-Institut für Biophysikalische Chemie, Abteilung Spektroskopie, \\ Am Fassberg, D-3400 Göttingen, Federal Republic of Germany
}

Reccived 30 August 1990

\begin{abstract}
Flash-excitation of degassed solutions of 2-(2'-hydroxyphenyl $)$ benzothiazole (HBT) in an inert solvent leads to the metastable triplet state of HBT and to a cis $\rightarrow$ trans isomerization of the HBT keto tautomer. Both processes proceed from the first excited singlet state of the HBT keto tautomer, ${ }^{1} \mathrm{~K}^{*}$. The trans-keto tautomer is not formed below $150 \mathrm{~K}$, whereas the triplet yield increases at lower temperatures, like the fluorescence yield. Besides fluorescence, intersystem crossing and cis $\rightarrow$ trans isomerization, an additional deactivation channel of ${ }^{1} K^{*}$ is proposed in order to explain the different temperature dependencies of the quantum yields of fluorescence and cis $\rightarrow$ trans isomerization. It is suggested that in the singlet ground state the keto-trans isomer decays by a second-order reaction to the enol form, ${ }^{1} \mathrm{E}$, by mutual hydrogen exchange: $2^{1} \mathrm{~K}_{\mathrm{tr}} \rightarrow 2^{1} \mathrm{E}_{\mathrm{tr}} \rightarrow 2^{1} \mathrm{E}_{\mathrm{cis}}$.
\end{abstract}

\section{Introduction}

In the present Letter we investigate some aspects of photoinduced proton or hydrogen transfer in 2 ( 2 '-hydroxyphenyl) benzothiazole (HBT). Since the photophysical behavior of HBT is in part similar to that of 2-(2'-hydroxyphenyl) benzoxazole (HBO), it is advantageous first to summarize briefly the photophysical properties of $\mathrm{HBO}$ in a non-polar solvent [1-3]. In the singlet ground state, the enol form, ${ }^{1} \mathrm{E}$ (HBO), is the stable tautomer. In the first excited singlet state, ${ }^{1} \mathrm{E}^{*}(\mathrm{HBO})$, rapid adiabatic proton transfer takes place, yielding the keto tautomer in its first excited singlet state, ${ }^{1} \mathrm{~K}^{*}$ (HBO). The lifetime of ${ }^{1} \mathrm{~K}^{*}(\mathrm{HBO})$ is determined by three processes: Intersystem crossing to the keto triplet state (rate constant $\left.k_{1}\right),{ }^{1} \mathrm{~K}^{*}(\mathrm{HBO}) \rightarrow{ }^{3} \mathrm{~K}^{*}(\mathrm{HBO})$, fluorescence ( rate constant $k_{2}$ ) and radiationless decay to the keto singlet ground state (rate constant $k_{3}=(9 \pm 1) \times$ $10^{11} \exp \left(-E_{3} / R T\right) \mathrm{s}^{-1} ; E_{3}=15 \pm 1 \mathrm{~kJ} / \mathrm{mol}$ [1]). Intersystem crossing and fluorescence emission are barrierless reactions, and the sum of the two rate constants is $k_{1}+k_{2}=1.8 \times 10^{8} \mathrm{~s}^{-1}$. The quantum yield of intersystem crossing from the excited enol singlet to the metastable enol triplet state ${ }^{\prime} \mathrm{E}^{*}(\mathrm{HBO}) \rightarrow^{3} \mathrm{E}^{*}(\mathrm{HBO})$ is virtually zero because of the competing ultrafast proton transfer reaction. Thus, immediately after laser flash excitation, only the triplet state ${ }^{3} \mathrm{~K}^{*}(\mathrm{HBO})$ is populated, followed by the establishment of an equilibrium ${ }^{3} \mathrm{~K}^{*}(\mathrm{HBO}) \rightleftharpoons$ ${ }^{3} \mathrm{E}^{*}$ (HBO) between the tautomeric triplet states $[2,3]$. Since, by chance, these two states are isoenergetic [2], $\mathrm{HBO}$ emits dual phosphorescence.
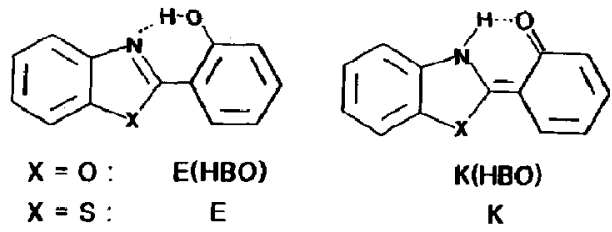

K(HBO)

K

Around room temperature the quantum yields $\Phi_{\text {ISC }}(T)=k_{1} /\left[k_{1}+k_{2}+k_{3}(T)\right]$ of intersystem crossing and $\Phi_{\mathrm{f}}(T)=k_{2} /\left[k_{1}+k_{2}+k_{3}(T)\right]$ of fluorescence are low because the value of $k_{3}(T)$ is large, whereas below $\approx 150 \mathrm{~K}$ the contribution of $k_{3}$ becomes negligible and $\Phi_{\mathrm{f}}$ and $\Phi_{\mathrm{ISC}}$ become constant.

The fluorescence properties of 2-(2'-hydroxyphenyl)benzothiazole (HBT) in non-polar solvents 
are quite similar to those of HBO. Excited state proton transfer ${ }^{1} \mathrm{E}^{*} \rightarrow{ }^{1} \mathrm{~K}^{*}$ is rapid $\left(1 / k_{\mathrm{PT}} \leqslant 5\right.$ ps [4], $1 / k_{\mathrm{PT}}=170 \pm 20 \mathrm{fs}$ [5]), and the fluorescence spectrum exhibits a large Stokes-shift because only the emission ${ }^{1} \mathrm{~K}^{*} \rightarrow{ }^{1} \mathrm{~K}$ of the keto tautomer is observed. The fluorescence quantum yield increases with decreasing temperature. According to several reports in the literature the triplet state of HBT was detected in flash experiments only under certain conditions. Nakagaki et al. [6] observed a $T_{L} \rightarrow T_{n}$ transition around $425 \mathrm{~nm}$ and a structured phosphorescence at $77 \mathrm{~K}$ only in the protic solvent mixture diethyl etherisopentane-ethanol (EPA) but not in non-polar solvents. Itoh and Fujiwara [7] observed in 3-methylpentane (MP) an oxygen-quenchable transient in absorption $\left(\lambda_{\max }=360 \mathrm{~nm}\right)$, which they tentatively assigned to a $T_{1} \rightarrow T_{n}$ transition "or some reaction products". The lifetime of another transient absorption between 420 and $480 \mathrm{~nm}$ was not affected by oxygen in the solution and was assigned to the keto tautomer in its singlet ground state. Becker et al. [8] questioned the assignment of the transient to the triplet state because in their flash experiments they could only detect transient absorptions that were not quenched by oxygen. Very recently, Brewer et al. [9] also observed a non-quenchable transient which absorbed with a broad maximum at $\lambda=452 \mathrm{~nm}$. In contrast to Itoh and Fujiwara they assigned this absorption to the trans isomer of the keto form, ${ }^{1} \mathrm{~K}_{\mathrm{tr}}$.

Most of these flash experiments were carried out at room temperature where, in analogy to $\mathrm{HBO}$, the intersystem crossing quantum yield is expected to be quite small and concealed by a reaction which does not occur with HBO. Therefore, we found it worthwhile to carry out a flash-photolytic investigation of HBT over a wide range of temperatures in a carefully dried and degassed non-polar solvent.

\section{Experimental}

HBT (Aldrich) was sublimed and recrystallized from absolute ethanol under nitrogen. $A$ few milligrams were purified further by HPLC. Both samples (colorless needles) had the same sharp melting point of $131.7-131.8^{\circ} \mathrm{C}$ (hot stage) (Lit.: $130-130^{\circ} \mathrm{C}[10]$, $131-132^{\circ} \mathrm{C} \quad[11], 131-131.5^{\circ} \mathrm{C}[12], 131.1-$ $131.7^{\circ} \mathrm{C}$ (corr.) $[13], 133.8-134.0^{\circ} \mathrm{C}$ (hot stage)
[14].) Cohen and Flavian [14], who reported the highest melting point, purified HBT on an alumina column and recrystallized it from aqueous ethanol. The use of aqueous ethanol gives higher yields, but recrystallization from absolute ethanol is a better purification method. Despite great efforts we could not achieve the melting point reported by Cohen and Flavian. Since the melting points of our two samples are equal and very sharp we presume that the value of Cohen and Flavian is too high.

The solvents 3-methylpentane (MP) (Jansen) and methylcyclohexane (MCH) (Fluka) were purified immediately before use on a silica-alumina column. Degassing and drying of the solutions were described in detail recently [3]. In order to carry out oxygenquenching experiments under comparable conditions and without getting moisture into the solution during the introduction of oxygen, a $50 \mathrm{ml}$ vessel containing dried oxygen was attached to the flash cell with a break seal. The flash call contained a solution of HBT which had been dried and degassed beforehand. After control experiments with the degassed solution the break seal was opened and the solution saturated with oxygen at room temperature by shaking. The partial oxygen pressure was roughly $0.5 \mathrm{~atm}$.

Fluorescence spectra were recorded with a Spex Fluorolog. The fluorescence cell $(10 \mathrm{~mm} \times 10 \mathrm{~mm}$ cross section) was housed in a quartz dewar with a copper block; the latter was cooled with a controllable stream of liquid nitrogen. Temperatures between 77 and $290 \mathrm{~K}$ could be maintained this way with an accuracy of $\pm 1.5 \mathrm{~K}$.

Laser flash experiments were carried out with an exciplex laser (Lambda Physik LPX 105) as described [3]. The time resolution was about 20 ns. For transient-decay times above $\approx 50 \mu$ s the pulsed $150 \mathrm{~W}$ Osram XBO lamp could be replaced by a 50 $\mathrm{W}$ tungsten halogen lamp as monitoring light source.

\section{Results}

\subsection{Temperature dependence of the fluorescence quantum yield}

In fig. 1 the temperature dependence of the fluorescence intensity, $I_{\mathrm{f}}(T)$, is shown. At all temperatures the fluorescence spectra exhibit only the strongly 


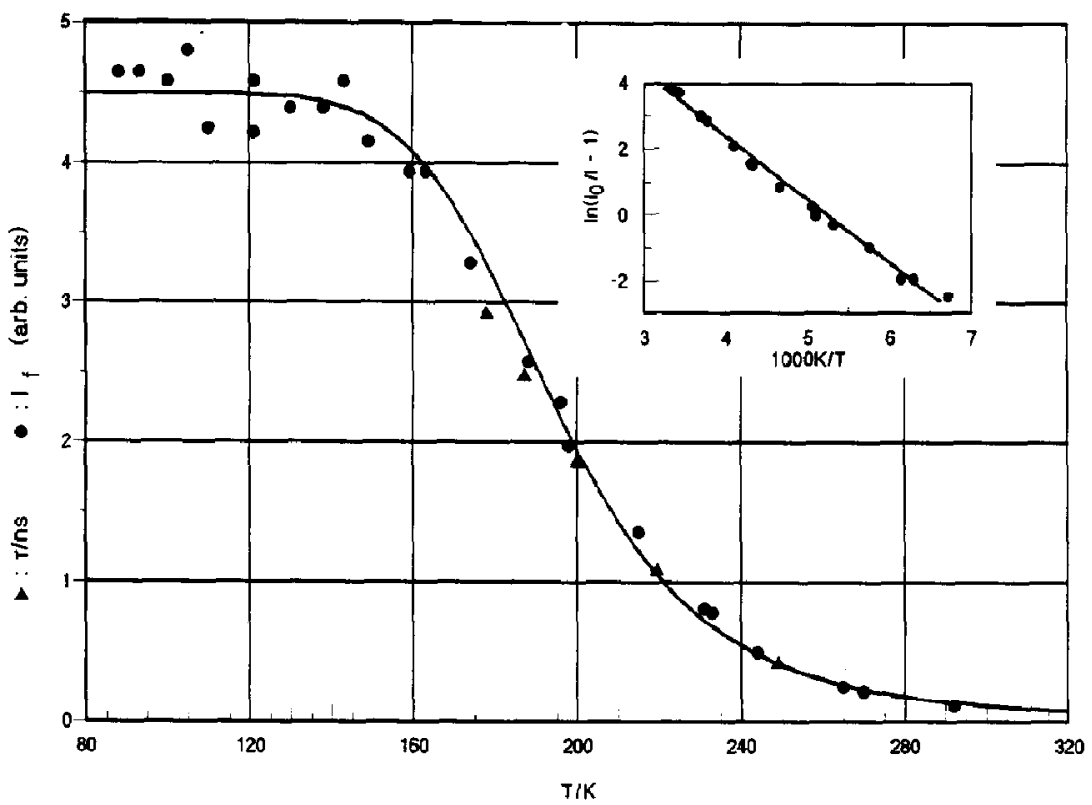

Fig. 1. Fluorescence intensity $I_{\mathrm{f}}\left(\boldsymbol{O}\right.$, arbitrary units) and fluorescence lifetime $\tau$ (ns) (A) of a degassed $5 \times 10^{-5} \mathrm{M}$ solution of HBT in MP. Excitation wavelength: $247 \mathrm{~nm}$. The solid line was calculated with $I_{\mathrm{f}}=A k_{1} /\left(k_{1}+k_{2}+k_{3}\right) . A k_{1}$ is a scaling factor, the values for the rate constants are the same as in table 1 . The frequency factor and the activation energy of $k_{3}$ were determined from the Arrhenius plot which is shown as insert (with $I_{0}=4.5$ units). The scaling of $I_{\mathrm{f}}$ was chosen in such a way that it had at $220 \mathrm{~K}$ the same value as the fluorescence lifetimes $\tau$ (ns) at that temperature.

Stokes-shifted fluorescence of the keto tautomer, ${ }^{1} \mathrm{~K}^{*} \rightarrow{ }^{1} \mathrm{~K}$, indicating that no hydrogen-bonding solvent impurities were present in the solution. Since the fluorescence bands gradually become narrower with decreasing temperature, the area of the spectra between 460 and $660 \mathrm{~nm}$ was taken as a measure of $I_{\mathrm{f}}(T)$. By lowering the temperature from 293 to 150 $\mathrm{K}, I_{\mathrm{f}}$ increases 30 -fold; below $150 \mathrm{~K}$ the fluorescence intensity is constant $\left(I_{\mathrm{f}}^{0}\right)$. Obviously, a thermally activated decay channel (rate constant $k_{3}=A_{3}$ $\left.\times \exp \left(-E_{3} / R T\right)\right)$ decreases the fluorescence yield at higher temperatures. By assuming that intersystem crossing $\left(k_{1}\right)$ and fluorescence $\left(k_{2}\right)$ are not thermally activated, one obtains that the fluorescence yield is $\Phi_{\mathrm{f}}(T)=k_{1} /\left[k_{1}+k_{2}+k_{3}(T)\right]$. Below $\approx 150$ $\mathrm{K}, \Phi_{\mathrm{r}}(T)$ becomes $\Phi_{\mathrm{f}}^{0}=k_{1} /\left(k_{1}+k_{2}\right)$. The fluorescence intensity ratio $I_{\mathrm{f}}^{0} / I_{\mathrm{f}}(T)$ depends on the temperature according to

$I_{\mathrm{f}}^{0} / I_{\mathrm{f}}(T)=1+\left[A_{3} /\left(k_{1}+k_{2}\right)\right] \exp \left(-E_{3} / R T\right)$.

A plot of $\ln \left[\left(I_{\mathrm{f}}^{0} / I_{\mathrm{f}}\right)-1\right]$ versus $1 / T$ (insert in fig. 1) yiclds the activation energy $E_{3}=16.2 \pm 0.5 \mathrm{~kJ} / \mathrm{mol}$ and the preexponential factor $A_{3}^{\prime}=A_{3} /\left(k_{1}+k_{2}\right)=$ $(3.0 \pm 0.7) \times 10^{4}$. The fluorescence lifetime $\tau(T)=$ $1 /\left[k_{1}+k_{2}+k_{3}(T)\right]$ shows the same temperature dependence as $I_{\mathrm{f}}(T)$ (cf. fig. 1 ) if one assumes that between 90 and $120 \mathrm{~K}$ it has the constant value $\tau_{0}=$ $1 /\left(k_{1}+k_{2}\right)=4.5 \pm 0.3 \mathrm{~ns}$, which is in good agreement with the value $\tau(\leqslant 100 \mathrm{~K}) \approx 5 \mathrm{~ns}$ in isopentane, reported by Barbara et al. [4]. With $A_{3}^{\prime}=$ $(3.0 \pm 0.7) \times 10^{4}$ and $\tau_{0}=4.5 \pm 0.3 \mathrm{~ns}$ the frequency factor of $k_{3}$ becomes $A_{3}=A_{3}^{\prime} / \tau_{0}=(8 \pm 2) \times 10^{12} \mathrm{~s}^{-1}$.

\subsection{Transient absorption spectra of $\mathrm{HBT}$}

On flashing a dry and oxygen-free solution of HBT in MP at low temperatures ( $\leqslant 150 \mathrm{~K}$ ) one observes a transient absorption with two maxima at 375 and $500 \mathrm{~nm}$ and a

$r$ at $\approx 580 \mathrm{~nm}$ (fig. 2). The decay pattern of this transient depends on its concentration, as shown in fig. 3: At low excitation intensities the decay is nearly monoexponential (fig. 3a), at high intensities the decay is of mixed first and second order (fig. $3 b$ ). 


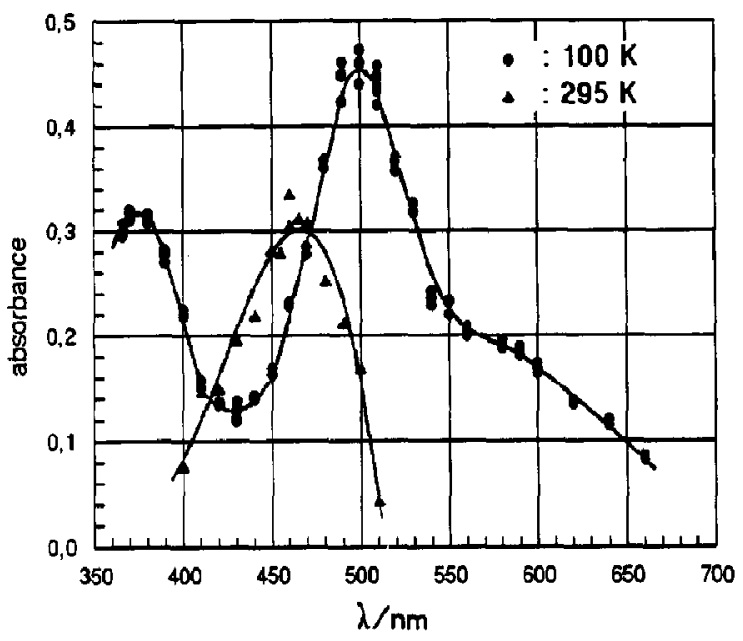

Fig. 2. Transient absorption spectra obscrved immediately after flashing a degassed solution of $1 \times 10^{-4} \mathrm{M} \mathrm{HBT}$ in MP at $298 \mathrm{~K}$ (A) and at $100 \mathrm{~K}$ (O) with the $248 \mathrm{~nm}$ line of an exciplex laser. The optical path length was $d=2 \mathrm{~cm}$.

On addition of oxygen to the solution this transient is quenched, that is, its initial absorbance remains unchanged, but its lifetime is strongly reduced (e.g. at $150 \mathrm{~K}$ from $100 \mu \mathrm{s}$ to $200 \mathrm{~ns}$ ) (fig. $3 \mathrm{c}$ ) and its decay becomes first order. This reduction in lifetime roughly corresponds to diffusion-controlled quenching of the transient species by oxygen and if the viscosity $(\eta)$ of the solvent is increased by lowering the temperature, the lifetime of the transient increases proportionally to $\eta / T$. At $100 \mathrm{~K}$, where the viscosity of MP is very high $\left(\eta(100 \mathrm{~K}) \approx 3 \times 10^{5} \mathrm{cP}[15]\right)$, the decay is first order also at high intensities and the lifetime of the transient is $1.5 \mathrm{~ms}$ (fig. 3d). Based on these observations we-assign this transient to the triplet state of HBT.

Above $\approx 170 \mathrm{~K}$ an additional transient is observed which has a broad maximum around $460 \mathrm{~nm}$. In agreement with the reports from other laboratories [6-9], we found that this transient is not quenched by oxygen. Since in an oxygen-saturated solution the lifetime of the oxygen-insensitive transient is much longer than the triplet lifetime, the two transients can be easily distinguished kinetically in such a solution. In oxygen-saturated as well as in degassed solution the decay of the transient absorbance $A(t)$ follows at low (fig. 3e) and high (fig. 3f) excitation intensities with high accuracy a second-order decay rate law with rate constants $k_{\mathrm{obs}}: \mathrm{d} A / \mathrm{d} t=-k_{\mathrm{obs}} A^{2}$. Since $k_{\mathrm{obs}}$ is determined from absorbance decay traces $(A(t)=$ $\epsilon c(t) d)$, its value $k_{\text {obs }}(T)=k_{\mathrm{s}}(T) / \epsilon d$ depends on the extinction coefficient $\epsilon$ of the transient at the monitoring wavelength and on the optical path length $d$ ( $k_{\mathrm{s}}$ is a second-order rate constant). If the viscosity of the solution is increased by lowering the temperature, $k_{\text {obs }}(T)$ increases according to $k_{\text {obs }}(T)=$ $F T / \eta$, where $F$ is a temperature-independent factor. This has been verified so far for two solvents: For MP between $200 \mathrm{~K}(\eta=1.5 \mathrm{cP})$ and $303 \mathrm{~K}(\eta=0.4$ $\mathrm{cP})$ and for $\mathrm{MCH}$ between $200 \mathrm{~K}(\eta=7 \mathrm{cP})$ and 327 $\mathbf{K}(\eta=0.7 \mathrm{cP})$. The yield of the non-quenchable transient is also temperature-dependent. It becomes gradually smaller at lower temperatures and drops to virtually zero below $150 \mathrm{~K}$ (cf. table 1 ).

\section{Discussion}

\subsection{The triplet state of $H B T$}

We believe that the assignment of the transient observed at low temperatures to the triplet state of HBT is unambiguous. The oxygen-quenching experiments, the dependence of the transient decay on the solvent viscosity and (in degassed solutions) on the excitation intensity provide the evidence for this assignment. We presume that the initial more rapid decay of the transient in fluid solution (cf. fig. 3b) is due to triplet-triplet annihilation (TTA) which is suppressed at $100 \mathrm{~K}$ because of the high viscosity of the solvent MP. As in the case of HBO [2], it should be possible to detect the delayed fluorescence resulting from TTA. Experiments in this direction are in progress. Because of the rapid proton transfer reaction in the excited singlet state, intersystem crossing takes place practically exclusively in the keto tautomer. Since at low temperatures the structured enol phosphorescence is only detectable in a protic solvent but not in a non-polar, glassy solution [6], we presume that, in contrast to $\mathrm{HBO}$, the enol triplet state of HBT lies energetically above the keto triplet state and that the former can therefore not be populated at low temperatures. At $100 \mathrm{~K}$ adventitious quenching is completely suppressed in MP and therefore ${ }^{3} K^{*}$ decays, like ${ }^{3} K^{*}(H B O)$, only by ra- 

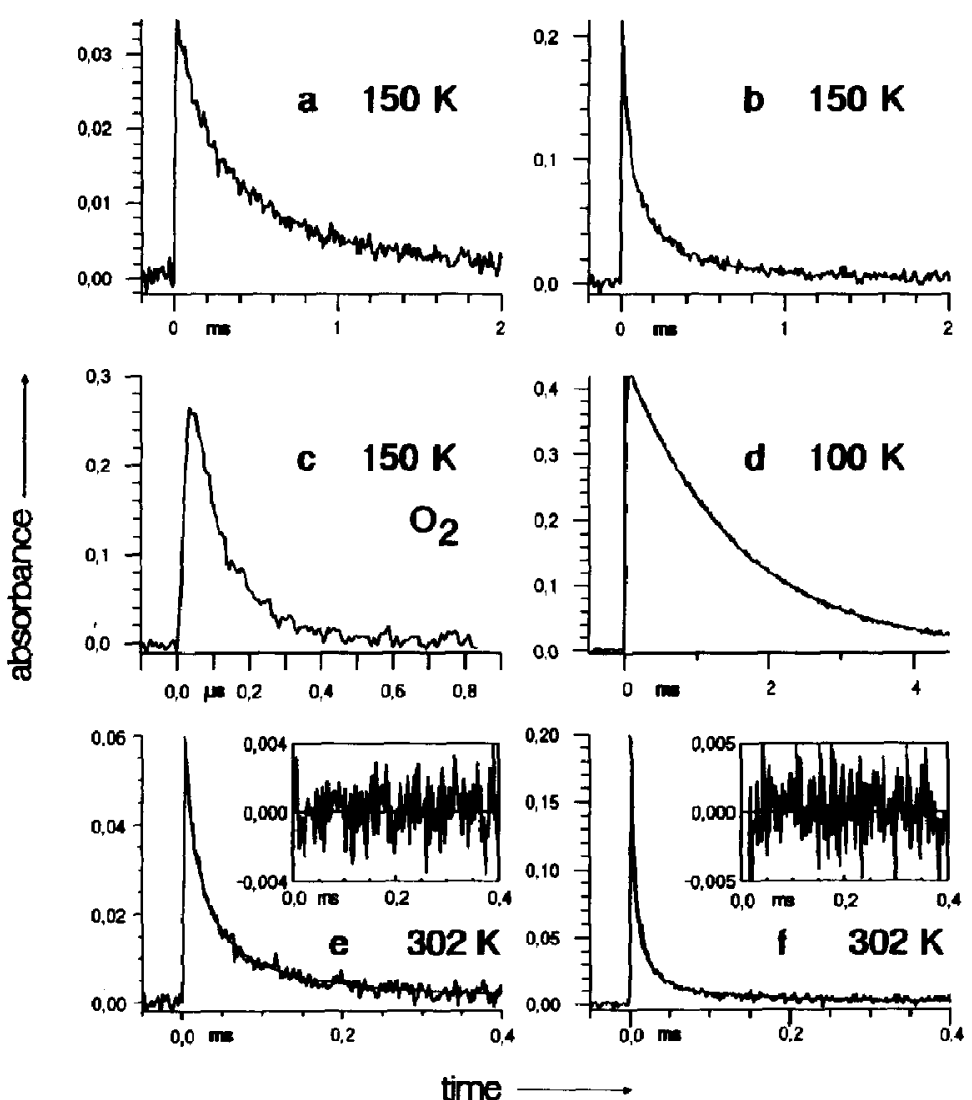

Fig. 3. Absorbance decay curves obtained after flashing a $1 \times 10^{-4} \mathrm{M}$ solution of HBT in MP at temperatures as indicated in the panels. The monitoring wavelength was $505 \mathrm{~nm}(d)$, and $460 \mathrm{~nm}(a-c, e, f)$. The solution was degassed ( $a, b, d-f$ ) or equilibrated with oxygen, partial pressure $\approx 0.5 \mathrm{~atm}$, (c). (a, e) Excitation with low intensity (note the absorbance scale). (d) Solid line: monoexponential fit to the observed trace. $(e, f)$ Solid lines: second-order fits to the observed traces, inserts: residuals in absorbance units.

diative and radiationless intersystem crossing to ${ }^{1} \mathrm{~K}$. The decay rate constant of ${ }^{3} \mathrm{~K}^{*}$ at $100 \mathrm{~K}$ in MP is with $670 \mathrm{~s}^{-1}$ about 3 times larger than that of ${ }^{3} K^{*}(\mathrm{HBO})$ under the same conditions $[2,3,16]$. Hence ${ }^{3} \mathrm{~K}^{*}$ lies presumably closer to the ground state than ${ }^{3} \mathrm{~K}^{*}(\mathrm{HBO})$, and therefore its phosphorescence origin will be located farther to the red than that of ${ }^{3} \mathrm{~K}^{*}(\mathrm{HBO})$.

\subsection{The non-quenchable transient}

The two-step-laser-excitation (TSLE) experiments of Brewer et al. [9] provide very convincing evidence that the oxygen-insensitive transient is not the cis isomer of the singlet ground state keto tau- tomer ${ }^{1} \mathrm{~K}_{\mathrm{els}}$, as proposed by Itoh and Fujiwara [7], because the TSLE emission is red-shifted with respect to the prompt fluorescence. Furthermore, the transient yield drops to zero below $150 \mathrm{~K}$ (cf. table 1). Brewer et al. [9] report that they could not detect any TSLE emission in methylcyclohexane at 77 $\mathbf{K}$ and that its yield decreased between room temperature and $77 \mathrm{~K}$. (The temperature dependence of the transient absorption is not discussed in ref. [9].) The yield of ${ }^{1} K_{\text {cis }}$ formation, however, has to become constant below $150 \mathrm{~K}$, like the fluorescence yield. Brewer et al. assign the non-quenchable transient $a b-$ sorption and the TSLE emission, which originates from the excitation of this transient, to the trans isomer of the keto form, ' $\mathrm{K}_{\mathrm{tr}}$, as shown below. This is, 
in our opinion, a very plausible assumption which we adopt for the discussion of our experimental results. We feel, however, that the interpretation of the absorbance decay curves in ref. [9] may be questioned.

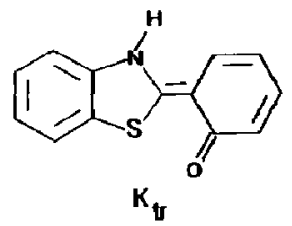

It is, of course, possible, to fit a biexponential decay function instead of a second-order law with satisfying accuracy to decay traces like those shown in fig. 3, because in this case four parameters (two amplitudes and two rate constants) are adjustable. As a tentative physical interpretation of such a fit to their absorption and TSLE-emission curves Brewer et al. [9] assume an equilibrium between a twisted conformer of the keto tautomer, ${ }^{1} \mathrm{~K}_{\mathbf{w}}$, and its trans isomer, ${ }^{1} K_{t r}$, accompanying the decay of the transient: ${ }^{1} \mathrm{~K}_{\mathrm{tw}} \rightleftharpoons{ }^{1} \mathrm{~K}_{\mathrm{tr}} \rightarrow{ }^{1} \mathrm{~K}_{\mathrm{cis}} \rightarrow{ }^{1} \mathrm{E}_{\mathrm{cis}}$. If this is the correct mechanism, one has to explain why the amplitude of the rapidly decaying component becomes larger with respect to the slow decay process when the laser excitation intensity is increased (cf. figs. $3 e$ and $3 f$ ).

An alternative and, in our opinion, more convincing interpretation of the absorbance decay is the assumption that the second-order fit to the decay traces (with two adjustable parameters) is appropriate. As mechanism we suggest (scheme 1) an intermolecular re-enolization process during which hydrogen bonds are formed between the $\mathrm{NH}$ and the $\mathrm{C}=\mathrm{O}$ groups of two keto trans-isomer molecules, followed by a mutual (concerted or consecutive) exchange of two hydrogen atoms whereby two molecules of the enol trans-isomer, ${ }^{1} E_{\mathrm{tr}}$, emerge, which quickly isomerize to the more stable cis form ' $\mathrm{E}_{\text {cis- }}$ Evidence for such a mechanism is, so far, the very accurate second-order fit to the decay curves of ${ }^{1} \mathrm{~K}_{\mathrm{tr}}$ and the dependence of $k_{\text {obs }}$ on the solvent viscosity and the monitoring wavelength. It is not possible to deduce from our values of $k_{\mathrm{obs}}$ whether the re-enolization reaction is diffusion-controlled $\left(k_{\mathrm{P}}>\right.$ $k_{\mathrm{d}}\left[{ }^{1} \mathrm{~K}_{\mathrm{tr}}\right]$, cf. scheme 1) or slower, because the extinction coefficient $\epsilon$ of ${ }^{1} K_{\mathrm{tr}}$ at the monitoring

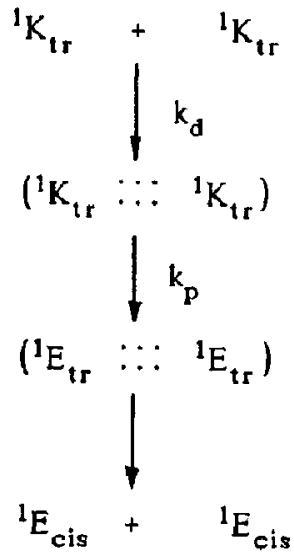

Scheme 1.

wavelength $\lambda_{\mathrm{m}}$ is not known. For instance, in $\mathrm{MCH}$ at $252 \mathrm{~K}$ and $\lambda_{\mathrm{m}}=462 \mathrm{~nm}, k_{\mathrm{obs}}=2.6 \times 10^{\mathrm{s}} \mathrm{s}^{-1}$, $\eta_{\mathrm{MCH}}(252 \mathrm{~K})=1.6 \mathrm{cP}$ and $k_{\mathrm{d}} \approx 8 R T / 3 \eta=3.5 \times 10^{9}$ $\mathbf{M}^{-1} \mathbf{s}^{-1}$. If the reaction is diffusion-controlled, i.e. $k_{\mathrm{s}}=k_{\mathrm{d}}=k_{\mathrm{obs}} \in d$, the extinction coefficient of ${ }^{1} \mathrm{~K}_{\mathrm{tr}}$ at $462 \mathrm{~nm}$ is (with $d=2 \mathrm{~cm}$ ) $\epsilon(462)=6700 \mathrm{M}^{-1} \mathrm{~cm}^{-1}$. Considering the structure of ${ }^{1} \mathrm{~K}_{\mathrm{tr}}$, this number could be quite close to the correct value, i.e. the re-enolization process may indeed be diffusion-controlled or close to it. This is, perhaps, not too surprising because the formation of just one hydrogen bond between two ${ }^{1} \mathrm{~K}_{\mathrm{tr}}$ molecules may suffice to prevent an efficient dissociation of the encounter complex and to accelerate the formation of a complex with the proper geometry for the mutual hydrogen exchange.

\subsection{Temperature dependence of fluorescence and cis $\rightarrow$ trans isomerization yields}

At first sight it appears plausible to assume that the cis $\rightarrow$ trans isomerization is the only reaction responsible for the altenuation of the fluorescence quantum yield at higher temperatures (cf. fig. 1). With this assumption the isomerization yield, $\Phi_{\mathrm{tr}}$, is $\Phi_{\mathrm{tr}}(T)=k_{3}(T) /\left[k_{1}+k_{2}+k_{3}(T)\right]$. It can be calculated with the rate constants whose values were determined from the $I_{\mathrm{f}}(T)$ data shown in fig. 1. In table 1 calculated ratios $R_{\text {calc }}=\Phi_{\mathrm{tr}}(T) / \Phi_{\mathrm{tr}}(327 \mathrm{~K})$ are listed together with measured absorbancies $A_{0}(T)$ and their ratios $R_{\text {obs }}=A_{0}(T) / A_{0}(327 \mathrm{~K})$. Since $R_{\text {obs }}$ decreases significantly more strongly with decreasing 
Table 1

$A_{0}(T)$ : absorbance observed immediately after flashing an oxygen saturated solution of $1 \times 10^{-4} \mathrm{M}$ HBT in MP at different temperatures, $T$; the monitoring wavelength was $460 \mathrm{~nm}$. $R_{\mathrm{obs}}=$ $A_{0}(T) / A_{0}(327 \mathrm{~K}) ; \quad R_{\text {call }}=\Phi_{\mathrm{tr}}(T) / \Phi_{\mathrm{tr}}(327 \mathrm{~K}), \quad \Phi_{\mathrm{tr}}=k_{3} /\left(k_{1}+\right.$ $k_{2}+k_{3}$ ) with $k_{1}+k_{2}=2.2 \times 10^{8} \mathrm{~s}^{-1}$ and $k_{3}=5 \times 10^{12}$ $\times \exp (-1950 \mathrm{~K} / T) \mathrm{s}^{-1} ; R_{\mathrm{calc}}^{\prime}=\Phi_{\mathrm{tr}}^{\prime}(T) / \Phi_{\mathrm{tr}}^{\prime}(327 \mathrm{~K}), \Phi_{\mathrm{tr}}^{\prime}=$ $k_{3} k_{3} /\left(k_{1}+k_{2}+k_{3}\right)\left(k_{4}+k_{5}\right)$ with $k_{4}=5 \times 10^{12} \mathrm{~s}^{-1}$ and $k_{5}=$ $10^{13} \exp (-722 \mathrm{~K} / T)$

\begin{tabular}{lllll}
\hline$T(\mathrm{~K})$ & $A_{0} \times 10^{3}$ & $R_{\mathrm{obs}}$ & $R_{\text {cslc }}$ & $R_{\text {calc }}^{\prime}$ \\
\hline 327 & 105 & 1 & 1 & 1 \\
302 & 110 & 1.05 & 0.99 & 0.85 \\
277 & 85 & 0.81 & 0.97 & 0.69 \\
250 & 70 & 0.67 & 0.93 & 0.51 \\
226 & 33 & 0.31 & 0.82 & 0.34 \\
201 & 14 & 0.13 & 0.60 & 0.17 \\
176 & 4 & 0.04 & 0.26 & 0.05 \\
\hline
\end{tabular}

temperatures than $R_{\text {calc, }}$ the activation barrier for the cis $\rightarrow$ trans isomerization must be higher than the barrier for the radiationless decay process which attenuates the fluorescence quantum yield.

Barbara et al. point out [4] that ${ }^{1} K^{*}$ may be vibrationally and torsionally excited and they propose that torsional motion about the $\mathrm{C}_{1}-\mathrm{C}_{7}$ bond of HBT is involved in the radiationless decay process. The comparison of $R_{\mathrm{obs}}$ with $R_{\mathrm{calc}}$ shows that at least two deactivation channels exist. A sequence of two steps (scheme 2) or two separate decay pathways from a common state (scheme 3 ) are conceivable. In scheme 2 , ' $\mathrm{K}_{\mathrm{tw}}$ is a necessary precursor of ${ }^{1} \mathrm{~K}_{\mathrm{tr}}$. The intermediate ${ }^{\prime} \mathrm{K}_{\mathrm{tw}}$ is a conformation of the keto tautomer where the angle $\varphi$ between the planes of the benzothiazole and the hydroxyphenyl moieties of HBT is $0^{\circ}<\varphi<90^{\circ}$. This torsional motion about the $\mathrm{C}_{1}-\mathrm{C}_{7}$ bond of HBT results in a loss of resonance stabilization energy, but at a certain angle $\varphi$ it could lead to a small minimum in the barrier between ${ }^{1} \mathrm{~K}_{\text {cis }}^{*}$ and ${ }^{1} \mathrm{~K}_{\mathrm{tr}}$, because at this angle the hydrogen bond between the $\mathrm{NH}$ and the $\mathrm{C}=\mathrm{O}$ group of ${ }^{1} \mathrm{~K}_{\mathrm{tw}}$ may be stronger than in the planar configuration of ${ }^{1} \mathrm{~K}_{\text {cis }}^{*}$ provided the NH group is not in the plane of the molecule. Within scheme 2 the isomerization quantum yield becomes

$\Phi_{\mathrm{tr}}^{\prime}=k_{3}(T) k_{5}(T) /\left[k_{1}+k_{2}+k_{3}(T)\right]\left[k_{4}+k_{5}(T)\right]$.

With the activation energies $E_{3}=16.2 \mathrm{~kJ} / \mathrm{mol}$ (cf. fig. 1) and the (fitted) value $E_{5}=6.0 \mathrm{~kJ} / \mathrm{mol}$ the ra-

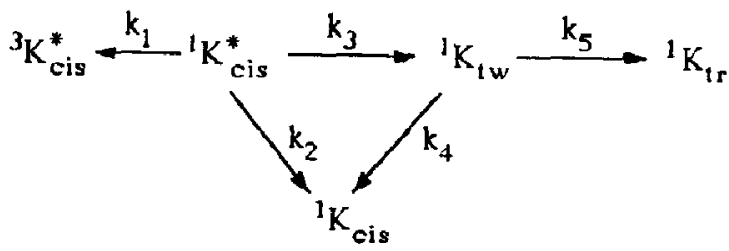

Scheme 2.

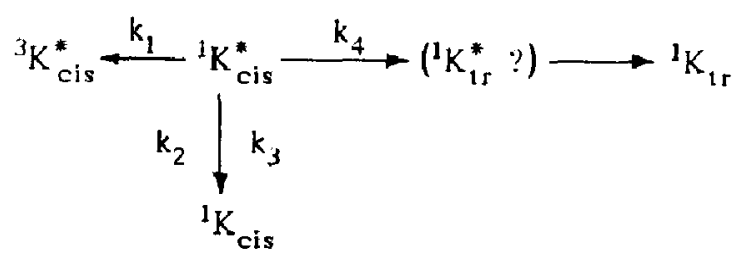

Scheme 3.

tios $R_{\text {calc }}^{\prime}=\Phi_{\mathrm{tr}}^{\prime}(T) / \Phi_{\mathrm{tr}}^{\prime}(327 \mathrm{~K})$ agree fairly well with $R_{\text {abs }}$ (cf. table 1 ).

In scheme 3 it is assumed that radiationless deactivation $\left(k_{3}(T)\right)$ and cis $\rightarrow$ trans isomerization $\left(k_{4}^{\prime}(T)=A_{4}^{\prime} \exp \left(-E_{4}^{\prime} / R T\right)\right)$ compete with each other. The Arrhenius plot in fig. 1 should not be linear in this case. Our $I_{\mathrm{f}}(T)$ data, however, are not accurate enough to distinguish between the two mechanisms because the difference between $E_{3}$ and $E_{4}^{\prime}$ is too small.

In the case of $\mathrm{HBO}$ the energy barrier $E_{5}$ (or $\left.E_{4}^{\prime}\right)$ may be 3-4 kJ/mol higher because the hydrogen bridge in ${ }^{1} \mathrm{~K}_{\text {cis }}^{*}(\mathrm{HBO})$ is stronger than in ${ }^{1} \mathrm{~K}_{\text {cis }}^{*}$ $[6,12]$. Such a slightly higher barrier reduces the yield $\Phi_{\text {Ir }}^{\prime}(\mathrm{HBO})$ so much that ${ }^{1} \mathrm{~K}_{\mathrm{rr}}(\mathrm{HBO})$ is already around $300 \mathrm{~K}$ not detectable in transient-absorption measurements. From this viewpoint, $\mathrm{HBO}$ and $\mathrm{HBT}$ have very similar photophysical properties, as one would expect, and only a small difference between $E_{5}(\mathrm{HBO})$ and $E_{5}$ (or between $E_{4}^{\prime}(\mathrm{HBO})$ and $E_{4}^{\prime}$ ) suffices to explain why a non-quenchable transient has not been detected in flash experiments with $\mathrm{HBO}$. It is, however, conceivable that the TSLE emission observed by Itoh and Fujiwara [7] is due to the generation of ${ }^{1} \mathrm{~K}_{\mathrm{tr}}(\mathrm{HBO})$, even if it is formed with small yield only, because the TSLE technique is more sensitive than direct absorbance measurements.

The cis-trans barrier in the ground state is most probably high. It would be interesting to investigate 
HBT in a solvent which has a high viscosity at temperatures above $180 \mathrm{~K}$ (where $\Phi_{\mathrm{tr}}^{\prime}$ is still high enough) in order to suppress the intermolecular reenolization reaction and to determine the lifetime of ${ }^{1} \mathrm{~K}_{\mathrm{tr}}$ under such conditions. It is also conceivable that cis $\rightarrow$ trans isomerization takes place in the triplet manifold (e.g. ${ }^{3} \mathrm{~K}_{\text {cis }}^{*} \rightarrow{ }^{1} \mathrm{~K}_{\mathrm{tr}}$ or ${ }^{3} \mathrm{~K}_{\text {cis }}^{*} \rightarrow{ }^{3} \mathrm{~K}_{\mathrm{tr}}^{*} \rightarrow{ }^{1} \mathrm{~K}_{\mathrm{tr}}$ ). Such processes should have an influence on the kinetics of the delayed fluorescence of HBT, which we are presently investigating.

\section{Acknowledgement}

We are greatly indebted to Dr. K. Zachariasse for performing the HBT fluorescence lifetime measurements with his single photon counting apparatus. We thank Dr. W. Kühnlc for the purification of HBT by HPLC and Mrs. A. Heinrich for technical assistance. We are grateful to the Deutsche Forschungsgemeinschaft (Sonderforschungsbereich 93 "Photochemie mit Lasern") for financial support.

\section{References}

[1] A. Mordziński and K.H. Grellmann, J. Phys. Chem. 90 (1986) 5503 .
[2] M.F. Rodriguez Prieto, B. Nickel, K.H. Grellmann and A. Mordiński, Chem. Phys. Letters 146 (1988) 387.

[3] K.H. Grellmann, A. Mordziński and A. Heinrich, Chem. Phys. 136 (1989) 201.

[4] P.F. Barbara, L.E. Brus and P.M. Rentzepis, J. Am. Chem. Soc. $102(1980) 5631$.

[5] F. Laermer, T. Elsaesser and W. Kaiser, Chem. Phys. Letters 148 (1988) 119.

[6] R. Nakagaki, T. Kobayashi and S. Nagakura, Bull. Chem. Soc. Japan 51 (1978) 1671.

[7] M. Itoh and Y. Fujiwara, J. Am. Chem. Soc. 107 (1985) 1561.

[8] R.S. Becker, C. Lenoble and A. Zein, J. Phys. Chem. 91 (1987) 3509.

[9] W.E. Brewer, M.L. Martinezz and P. Chou, J. Phys. Chem. 94 (1990) 1915.

[10] K. Anthony, R.G. Brown, J.D. Hepworth, K.W. Hodgson, B. May and M.A. West, J. Chem. Soc. Perkin Trans. II (1984) 2111.

[11] A. Cerniani and P. Passerini, J. Chem. Soc. (1954) 2261.

[12] J. Durmis, M. Karvas and Z. Manasek, Coll. Czech. Chem. Commun. 38 (1973) 215.

[13] M.T. Bogert and H.B. Corbitt, J. Am. Chem. Soc. 48 (1926) 783.

[14] M.D. Cohen and S. Flavian, J. Chem. Soc. B (1967) 317.

[15] B. Nickel and $H$. Lesche, unpublished results.

[16] B. Nickel and M.F. Rodrigues Prieto, Chem. Phys. Letters 146 (1988) 393. 